Service social

\title{
Le travail social en 1990 : de la « transition permanente »
}

\section{Gilles Tardif}

Volume 35, numéro 3, 1986

Les jeunes et le travail social

URI : https://id.erudit.org/iderudit/706311ar

DOI : https://doi.org/10.7202/706311ar

Aller au sommaire du numéro

Éditeur(s)

École de service social de l'Université Laval

ISSN

1708-1734 (numérique)

Découvrir la revue

Citer ce document

Tardif, G. (1986). Le travail social en 1990 : de la « transition permanente ».

Service social, 35(3), 283-289. https://doi.org/10.7202/706311ar d'utilisation que vous pouvez consulter en ligne.

https://apropos.erudit.org/fr/usagers/politique-dutilisation/ 


\section{ÉDITORIAL}

TARDIf, Gilles, Secrétaire général du Bureau de consultation jeunesse de Montréal.

\section{Le travail social en 1990 : de la "transition permanente" 1}

Je ne suis qu'un gestionnaire d'un organisme communautaire pour les jeunes; je ne suis pas théoricien, ni chercheur, ni homologué d'un titre quelconque; j'ai la seule prétention de m'impliquer dans les ressources communautaires et de m'obstiner à y développer des idées, des actions auprès ou avec les jeunes. Je tente ici d'exposer certaines réflexions et commentaires sur les jeunes et le travail social que vous prendrez comme bon vous semble; mais je souhaite tout de même que mes propos vous portent à réfléchir.

S'il faut parler d'espoir et d'horizons nouveaux pour la jeunesse, il faut alors mettre en relief la remise en question du travail social des dernières années : reconnaître, par exemple, que le travail social situe l'intervenant de l'autre côté de la barrière par rapport aux clients; reconnaître que l'intervenant est porteur, dans son travail, d'idéologies, et que les dernières législations s'alimentent d'idées surtout très concrètes au plan de la gestion sociale; s'apercevoir que les alliances portant sur la misère ou sur l'intervention alternative n'ont pas porté de fruits et qu'elles n'ont, au plus, que permis l'identification des opposants. Malgré toute sa bonne volonté, la position qu'occupe l'intervenant et son appartenance à l'entreprise sociale le placent en rupture de classe, de sorte que ses désirs de médiation ou de négociation ont peu de portée. Même l'idée de développer « des travailleurs sociaux aux pieds nus » ne permet pas de faire éclater le fonctionnarisme social. Bien plus, c'est par les jeunes et leurs démêlés avec l'aide sociale qu'on a pu 
constater que la pauvreté permet de faire de ces derniers des otages de l'État.

Je reprendrais ici l'expression de Frédérik Mispelbloom ${ }^{2}$ pour qualifier l'année 1985, Année internationale de la jeunesse, comme un symptôme positif pour les jeunes. Cette année se voulait une remise en question de l'ordre établi, mais elle est restée prisonnière des idées qu'elle mettait en question : en conséquence, les groupes de jeunes et les organismes se sont mutuellement neutralisés.

J'oserais dire que les jeunes actuels sont invulnérables, parce qu'ils réussissent à créer et à bâtir à partir de presque rien. La difficulté d'adaptation des jeunes à la société actuelle dépasse de beaucoup celle de la génération précédente. Je cite souvent cet exemple de travailleurs sociaux qui, dans une session de formation commandée par le C.S.S.M.M., disaient être affectés par leur travail, se sentant détestés et mal aimés, et chercher un autre type de relation avec les jeunes. Je relie cela à une enquête du Ministère de l'éducation québécois ${ }^{3}$ portant sur les valeurs des jeunes, qui rapportait qu'un fort pourcentage de jeunes recherchaient l'amitié, une relation sentimentale dans une société où ils subissaient le détachement de leur aînés et des intervenants : curieuse jonction où l'on pourrait croire que les uns finiront par comprendre les autres et questionner leur travail. Je ne crois pas que le travailleur social ait trouvé réponse à ce problème sinon en étant cynique et plus fonctionnel ; mais il faut s'étonner des réponses des jeunes qui vivent, dans la rue, la nuit, de cette quête de relations plus humaines. Ils ont, semble-t-il, compris que le travailleur social n'est pas la solution à leurs problèmes et, qu'en tant que clients, ils existent parce qu'ils en consomment des services, tout en sachant très bien que les services "ne sont pas de service". C'est ce que notre organisme, le Bureau de consultation jeunesse (B.C.J.) déclarait, le 27 novembre 1984, dans La Presse : ${ }^{4}$ beaucoup de jeunes "décrochent des services" pour éviter d'être piégés et d'être atteints dans leur intelligence et leur sensibilité : espoir certain que cette résistance à l'abrutissement des institutions! La déviance des jeunes marginaux se poursuit dans une solidarité réelle. Échec du volontariat de certaines mesures, échec des mesures préventives, développement de réseaux d'aide et de travail au noir tout autant que de l'universalisation de la sexualité, de la drogue (le tiers des jeunes de moins de 17 ans ont des relations sexuelles) ${ }^{5}$... Ce type de comportement attaque les valeurs des adultes et fait reculer plus d'un intervenant ; il représente en tout cas un défi certain pour notre société. Je ne pense pas qu'il s'agisse d'un agir conscient chez les jeunes, mais plutôt des retombées de notre inconscience à vouloir gérer le social comme une chose, alors que les jeunes, entre autres, nous obligent à considérer leur 
vécu : défi pour notre société qui, déjà, articule sa tendance à mater cette jeunesse "perdue " plutôt que d'y reconnaître une forme d'adaptation aux difficultés qu'elle éprouve.

Ma pratique dans le réseau communautaire est souvent en opposition au travail social et je ne peux écrire ce texte sans conseiller au lecteur d'être prudent. Je voudrais cependant souligner ici deux anecdotes. J'étais étonné de lire, récemment, dans un rapport du Comité sénatorial sur la jeunesse, ${ }^{6}$ ce commentaire d'un représentant de l'Armée du salut qui disait: "C'est différent, je ne sais pas quoi, mais je sens quelque chose de différent... une force évangélique vraie qui se traduit par du travail social pratique [...] parce qu'on aime les gens. " Mais étonné, je l'étais déjà quand j'ai entendu un vieil ami me raconter comment, en allant rendre visite à un groupe d'alcooliques anonymes, il avait été impressionné parce qu'on lui avait offert de le ramener chez lui après la soirée : "On s'est occupé de moi", disait-il, "même à l'Église, ça ne se fait plus». Je dois avouer que, pour moi, ces deux petites histoires expliquent un peu ce qui manque dans notre société. Le défi à relever consiste à s'occuper des gens, malgré une absence de motifs religieux ou " anonymes" pour motiver la pratique, et même si on n'a pas d'idée de ce qu'on fait. Les groupes de pairs semblent aborder cet aspect de la relation dans l'aide, mais il y manque le sens des gestes. À mon avis, quand on fait appel au civisme, à la responsabilitè du citoyen, et c'est le cas dans l'entraide, il faut oublier le client. Un client attend un service, un citoyen offre ses services. Le travailleur social qui propose l'entraide est subversif dans l'ordre actuel des choses, mais si le sens lui échappe, il ne fait alors qu'ajouter aux alternatives, il perd le défi de mettre l'accent sur le sens que peut avoir l'entraide comme façon de dire autrement le vécu social. En fait, le défi conduit à mener les intervenants sociaux à redécouvrir le monde, reconcevoir le travail social, avant que l'Institut C.D. Howe et son directeur en tête, M. Claude Forget, ne le fassent pour tous. Au printemps 1985, dans une rencontre organisée par Centraide Montréal, Monsieur Forget a bel et bien annoncé que, devant l'échec du discours social des années 1960 et 1970 et la crise occidentale, il fallait reformuler plus scientifiquement ce discours pour l'adapter aux années 1980 et relever le défi de remodeler la conscience de l'intervention sociale future pour qu'elle ne soit pas idéologique, mais pratique et concertée. Comme l'a déjà dit Aristote, le monde social peut être construit et dit de plusieurs façons; le seul problème est de préserver un espace où le débat politique sur le monde social puisse se tenir. Il ne s'agit pas de mettre les marginaux dans des réserves, comme on l'a fait avec les autochtones depuis plus de cent ans, ou de maintenir des peuples sous l'emprise coloniale tels les territoires du Nord-Ouest, 
mais plutôt d'envisager, avec les acteurs sociaux, un monde où chacun, à partir de sa position sociale, réussit à construire un monde social où ily a lutte contre l'injustice, puisque toute injustice qui frappe l'homme dévalorise l'Humanité.

Au Québec, le dossier jeunesse a été marqué d'enquêtes et de rapports dénonçant des injustices (rapport Batshaw, ${ }^{7}$ rapport du Conseil supérieur de l'éducation ${ }^{8}$ ), mais on s'est empressé d'oublier les dénonciations pour mettre en place une série de systèmes garantissant, soidisant, une intervention plus juste. Or, chacun sait qu'il n'en est rien et que, bien qu'on ait fait taire les dénonciations, les injustices se perpétuent. On n'a qu'à lire le récent rapport de Centraide Montréal ${ }^{9}$ sur les réalités sociales du Montréal métropolitain pour réaliser que notre système a un prix à payer qui n'est pas que symbolique. Le sacrifice de générations de jeunes fait partie d'un rituel qui pourrait permettre à notre société de participer au bien-être occidental et postindustriel, de se prémunir contre une mort sociale dont le suicide de plusieurs jeunes serait un indice prophétique. À moins que, parmi nous, s'en glissent certains qui ne veulent plus payer pour ce que ce système procure, qui voudraient rompre avec cet état de misère ou substituer une autre voie à ces "désordres de la dépendance ". Nous sommes en effet dépendants les uns des autres et cela devrait appeler à la solidarité et non à la méfiance : savoir lire nos dépendances plutôt que les développer au détriment d'une partie de la société. Dans la mesure où le service social est devenu une partie de l'administration publique, sa marge de manœuvre se restreint et l'empêche de négocier l'état présent et à venir de la société. Dans le dossier jeunesse, la recherche sociale semble beaucoup trop à la remorque de données américaines et pas assez développée sur nos propres bases; après tout, nos corporations, associations, écoles et facultés peuvent soumettre des idées et des projets pour réduire l'ignorance et la faiblesse dramatique de la pensée sociale. Elles pourraient suggérer que l'évolution rapide, au Canada, des méthodes de prévention du crime basées sur des données socio-historico-familiales vienne confirmer la conception arrêtée qu'on a d'une société plutôt que d'essayer une nouvelle méthodologie d'intervention fondée sur des contextes différents et des populations variées qui composent le tissu social.

Mais il semble que, frappé par les difficultés et les échecs du travail social, on décide généralement de changer son style de vie plutôt que ses actions. Par exemple, à défaut d'agir conformément aux droits, on préfère se retrancher du côté des responsabilités unilatérales, et on laisse de côté tout l'aspect de la participation des usagers, ce qui pourrait être un champ d'implication des jeunes plutôt que d'en faire 
des bibelots de circonstance. D'une autre manière, les syndicats, plutôt que d'être une force importante, se contentent de suivre la bureaucratisation et négligent toutes les stratégies qui aboutiraient à des alliances dans la négociation des pratiques. Ce type d'approche, s'il était encouragé, permettrait de considérer l'éveil d'une solidarité dans le réel avec les jeunes; il permettrait de ne plus ignorer certaines blessures qui rendent la vie impossible; il amènerait certains à construire une mise au monde qui dépasse la parole, une mise au monde à partir de choses simples et banales, mais combien exigeantes quand elles s'attachent à la vie quotidienne et qu'elles obligent à un engagement direct auprès des jeunes.

C'est dans cet engagement qu'on est alors forcé de reconnaître que plusieurs valeurs se sont modifiées et qu'il est important d'en saisir l'actualisation. À titre d'exemple, disons que, pour plusieurs jeunes, "tripper", ça peut vouloir dire "tenter de trouver un sens à ce qui est vécu dans un contexte d'essais-erreurs". Les notions de travail et de temps ont changé. Plusieurs d'entre nous avons "trippé "à acquérir un certain confort matériel, mais beaucoup de jeunes cherchent autre chose; ils veulent vivre intensément, devenir autonomes: vivre "au boutte" dans une société où l'espace est réduit. II veulent créer des liens, briser leur isolement dans un monde d'individualités, dans un monde où les emplois sont rares et l'avenir incertain. Les adultes sont invités à être corrects avec les jeunes, c'est-à-dire cohérents et authentiques; c'est là que le style de vie de plusieurs d'entre eux compromet la possibilité de contact. L'attitude des intervenants face aux jeunes est implicite alors qu'elle doit être explicite. La vérité des engagements est un enjeu des luttes à mener pour légitimer les valeurs et la vie qu'on mène. Je soulignais qu'à partir du moment où on reconnaît qu'on occupe une position sociale, il devrait être impérieux de se familiariser avec les autres positions occupées par d'autres personnes ou groupes afin de prendre position; et les jeunes, actuellement, par leurs styles et leurs valeurs, nous invitent à cet effort de reconnaissance. Je fais ici référence à un récent colloque (colloque Plus que possible, 22-24 novembre 1985, R.O.C.J.M.M. ${ }^{10}$ ) où des jeunes, ayant pris position dans les débats, occupaient une scène privilégiée face à des intervenants encore embêtés par leurs hésitations, leurs questions idéologiques, leurs scrupules personnels. Malheureusement pour les jeunes, le débat s'est avéré décevant puisque, visiblement, la mobilisation reste une pensée et ne va pas jusqu'à vouloir modifier les facteurs qui confèrent leur sens aux enjeux sociaux. Autrement dit, sans être trop gauchisant, beaucoup d'intervenants sont à cheval entre la culture qui les porte et la contre-culture qui les déclasse. D’ailleurs, ces jeunes ne demandent pas 
nécessairement qu'on leur donne le droit de faire, mais plutôt qu'on leur donne accès aux moyens de faire. Plusieurs ont appris, par la force des choses, à se débrouiller et, de ce fait, ne sont plus " aidables "; si l'on admet que l'objet du travail social est l'inégalité des droits, on finit par arriver à déplacer la relation aidant-aidé en celle d'aidé-aidant où l'un et l'autre s'entraident à réaliser ce qui ne peut être fait seul : se mettre au monde. L'aide est un droit et non un pouvoir, une dépendance, ou une charité et elle suppose une solidarité réciproque et vécue, où chacun est auteur de sa manière de vivre en dépit des contraintes qui pèsent sur lui.

La politique de services développée au Québec a nié notre pauvreté pour nous faire accéder à une plus forte domesticité. II semble que les récentes lois, qui repoussent des phénomènes comme la prostitution ou la drogue, contribuent à nous soumettre socialement tout en nous donnant l'illusion d'être libres. Je crois, peut-être de façon utopique, que cette jeunesse qui résiste à la domestication et qui, instinctivement, se regroupe, est plus saine que nous. Nous avons abjuré notre bon sens en tolérant des écoles telles que nous les connaissons; les jeunes en décrochent et je ne vois pas comment on peut intelligemment les forcer à y retourner sans opérer de changement. Pour les jeunes, le travail est une notion différente puisqu'ils n'acceptent pas l'état d'esprit qui le modèle. Il n'y a plus de salut par le travail, comme il n'y a pas de salut par le travail social. C'est dommage pour tous ceux qui en vivent, mais les travailleurs sociaux du Québec sont porteurs de domestication et, pour s'y opposer, ils doivent refuser socialement d'y travailler. En même temps, ce geste ne peut être fait sans être porté par une idée d'une société différente. L'Année internationale de la jeunesse voulait nous sensibiliser et nous faire contester l'ordre actuel des choses, mais tel ne fut pas le cas; les problèmes restent entiers et le pire que nous puissions faire, c'est de les repasser aux jeunes: ça, ça n'aurait pas de sens.

\section{Notes et références}

1 Expression tirée de : Umberto Eco, La Guerre du faux, Paris, Grasset, 1985.

2 Mispelbloom, F., dans: Cahiers de pratiques sociales, 1985.

3 Ministère de l'éducation du Québec, Les valeurs des jeunes de 16 à 20 ans, Québec, Éditeur officiel du Québec, 1980.

4 Bernier, C., "Plusieurs jeunes refusent l'aide médicale ou sociale ", La Presse, 27 novembre 1984, p. A-10.

5 Tessier, M., Sexualité et prévention : d'abord l'affaire des jeunes, Montréal, Bureau de Consultation jeunesse, 1985. 
6 Comité sénatorial sur la jeunesse, Jeunesse : un plan d'action, Ottawa, 1986.

7 Ministère des affaires sociales du Québec, Rapport du Comité d'étude sur la réadaptation des enfants et adolescents placés en centre d'accueil, Québec, Bibliothèque nationale du Québec, 1975.

${ }^{8}$ Conseil supérieur de l'éducation, Pour que les jeunes adultes puissent espérer : avis sur la formation des jeunes adultes défavorisés et leur insertion sociale et professionnelle, Québec, Gouvernement du Québec, Direction des communications, 1983.

9 Centraide Montréal, Les besoins de la communauté, Montréal, Centraide Montréal, 1986.

10 Regroupement des organismes communautaires jeunesse de Montréal, Actes du colloque "Plus que possible », Montréal, R.O.C.J.M., 1985. 